\title{
On a certain impulsive differential system with piecewise constant arguments
}

\author{
Mehtap Lafci · Huseyin Bereketoglu
}

Received: 28 February 2014/Accepted: 23 April 2014/Published online: 21 May 2014

(c) The Author(s) 2014. This article is published with open access at Springerlink.com

\begin{abstract}
We study the existence of periodic solutions of a first order nonlinear impulsive differential system with piecewise constant arguments.
\end{abstract}

Keywords Carvalho's method · Periodic solution · Impulsive differential system · Piecewise constant arguments

\section{Introduction}

In the past two decades, the theory of impulsive differential equations has been developed very rapidly. Such equations consist of differential equations with impulse effects and emerge in modelling of real-world problems observed in engineering, physics and biology, etc. The books [1-3] are good sources for the study of impulsive differential equations and their applications. In addition to these, there exist many papers that investigate the behaviour of solutions of impulsive differential equations [4-8].

Since the early 1980s, differential equations with piecewise constant arguments have attracted great deal of attention of researchers in science. Differential systems with piecewise constant arguments appear in diverse areas such as engineering, physics and mathematics. The work [9] covers a systematical study on mathematical models with piecewise constant arguments. Differential equations with piecewise constant arguments are closely related to

M. Lafci $(\bowtie) \cdot$ H. Bereketoglu

Department of Mathematics, Faculty of Sciences, Ankara

University, Tandogan, 06100 Ankara, Turkey

e-mail: mlafci@ankara.edu.tr

H. Bereketoglu

e-mail: bereket@science.ankara.edu.tr difference and differential equations. Therefore, they are stated as hybrid dynamical systems [10]. The qualitative works on oscillation, periodicity and convergence of solutions of differential equations with piecewise constant arguments have been done by works [11-19]. Also, Wiener's book [20] is a distinguished source with respect to this area.

But, there are only a few papers [21-23] for impulsive differential equations with piecewise constant arguments.

Moreover, in [24], Seifert has taken into consideration the scalar equation

$x^{\prime}(t)=\lambda x(t)-g(x[t])$

which shows a continuous dynamical system and proved that this equation has a periodic solution with period 2 .

So, we have been motivated consider the impulsive differential system with piecewise constant arguments

$$
\left\{\begin{array}{l}
x_{1}^{\prime}(t)=\lambda x_{1}(t)-g\left(x_{2}[t-1]\right), \\
x_{2}^{\prime}(t)=\lambda x_{2}(t)-g\left(x_{1}[t-1]\right), \quad t \neq k \in \mathbb{Z}^{+}=\{1,2, \ldots\}, t \geqslant 0,
\end{array}\right.
$$

$x_{1}\left(t^{-}\right)=d x_{1}(t), \quad x_{2}\left(t^{-}\right)=d x_{2}(t), \quad t=k \in \mathbb{Z}^{+}$,

where $\lambda>0$ is a real constant, $d \in \mathbb{R} \backslash\{0,1\}, g: \mathbb{R} \rightarrow \mathbb{R}$ is a continuously differentiable function, $x_{1}\left(k^{-}\right)=\lim _{t \rightarrow k^{-}} x_{1}(t)$, $x_{1}(k)=x_{1}\left(k^{+}\right)=\lim _{t \rightarrow k^{+}} x_{1}(t), x_{2}\left(k^{-}\right)=\lim _{t \rightarrow k^{-}} x_{2}(t) \quad$ and $x_{2}(k)=x_{2}\left(k^{+}\right)=\lim _{t \rightarrow k^{+}} x_{2}(t)$, i. e., $x_{1}(t)$ and $x_{2}(t)$ are right continuous at $t=k$ and [.] denotes the greatest integer function.

It is noted that (1)-(2) is a discontinuous dynamical system which may be regarded as a competition model of two species competing for the same resources. 
Our aim is to study the existence of solutions of (1)-(2) and search periodic solutions with period 2 using Carvalho's method which is given below.

Theorem 1 (Carvalho's method, [25]) If $p$ is a positive integer and $x(k)$ is a periodic sequence of period $p$, then the following hold true:

(i) If $p>1$ is odd and $m=\frac{p-1}{2}$, then

$$
x(k)=a_{0}+\sum_{j=1}^{m} a_{j} \cos \left(\frac{2 j k \pi}{p}\right)+b_{j} \sin \left(\frac{2 j k \pi}{p}\right),
$$

for all $k \geqslant 1$.

(ii) If $p$ is even and $p=2 m$, then

$$
x(k)=a_{0}+a_{m} \cos \pi k+\sum_{j=1}^{m-1} a_{j} \cos \left(\frac{2 j k \pi}{p}\right)+b_{j} \sin \left(\frac{2 j k \pi}{p}\right),
$$

for all $k \geqslant 1$.

For example, if $p=2$, then

$x(k)=a_{0}+a_{1} \cos \pi k$.

A solution of (1)-(2) is defined as below.

Definition 2 A function $\left(x_{1}(t), x_{2}(t)\right)$ defined on $[0, \infty)$ is said to be a solution of (1)-(2) if it satisfies the following conditions:

1. The components $x_{1}, x_{2}:[0, \infty) \rightarrow \mathbb{R}$ are continuous for $t \in[0, \infty)$ with the possible exception of the points $[t] \in(0, \infty)$,

2. $\left(x_{1}(t), x_{2}(t)\right)$ is right continuous and has left-hand limits at the points $[t] \in(0, \infty)$,

3. $\left(x_{1}^{\prime}(t), x_{2}^{\prime}(t)\right)$ exists for every $t \in[0, \infty)$ with the possible exception of the points $[t] \in[0, \infty)$ where one-sided derivates exist,

4. $\left(x_{1}(t), x_{2}(t)\right)$ satisfies system (1) on each interval $k<t<k+1, k \in \mathbb{N}=\{0,1,2, \ldots\}$,

5. $x_{1}(t)$ and $x_{2}(t)$ satisfy, respectively, (2) at $t=1,2, \ldots$

\section{Main results}

We prove the following results:

Theorem 3 Any solution $\left(x_{1}(t), x_{2}(t)\right)$ of (1)-(2) on the interval $[0, \infty)$ has the following form:

$$
\left\{\begin{array}{l}
x_{1}(t)=x_{1}([t]) \exp \lambda(t-[t])-g\left(x_{2}([t-1])\right) \frac{\exp \lambda(t-[t])-1}{\lambda}, \quad t \neq k \in \mathbb{N}, \\
x_{2}(t)=x_{2}([t]) \exp \lambda(t-[t])-g\left(x_{1}([t-1])\right) \frac{\exp \lambda(t-[t])-1}{\lambda}
\end{array}\right.
$$

and for $t=k, k \in \mathbb{N},\left(x_{1}(k), x_{2}(k)\right)$ satisfies the difference system

$$
\left\{\begin{array}{l}
x_{1}(k+1)=\frac{\alpha}{d} x_{1}(k)-\frac{\beta}{d} g\left(x_{2}(k-1)\right), \\
x_{2}(k+1)=\frac{\alpha}{d} x_{2}(k)-\frac{\beta}{d} g\left(x_{1}(k-1)\right),
\end{array}\right.
$$

where $\alpha=\exp \lambda$ and $\beta=(\exp \lambda-1) / \lambda$.

Proof system (1), in the interval $k<t<k+1$, can be reduced to the ordinary differential equations system

$$
\left\{\begin{array}{l}
x_{1}^{\prime}(t)-\lambda x_{1}(t)=-g\left(x_{2}(k-1)\right), \\
x_{2}^{\prime}(t)-\lambda x_{2}(t)=-g\left(x_{1}(k-1)\right) .
\end{array}\right.
$$

Solving system (8), we get

$\left\{\begin{array}{l}x_{1}(t)=x_{1}(k) \exp \lambda(t-k)-g\left(x_{2}(k-1)\right) \frac{\exp \lambda(t-k)-1}{\lambda}, \\ x_{2}(t)=x_{2}(k) \exp \lambda(t-k)-g\left(x_{1}(k-1)\right) \frac{\exp \lambda(t-k)-1}{\lambda}, \quad k<t<k+1 .\end{array}\right.$

Replacing $k$ by $[t]$, we obtain (6).

Before applying the impulse condition at $t=k$, we also find the solution of system (1) in the interval $k-1<t<k$ as $\left\{\begin{array}{l}x_{1}(t)=x_{1}(k-1) \exp \lambda(t-k+1)-g\left(x_{2}(k-2)\right) \frac{\exp \lambda(t-k+1)-1}{\lambda}, \\ x_{2}(t)=x_{2}(k-1) \exp \lambda(t-k+1)-g\left(x_{1}(k-2)\right) \frac{\exp \lambda(t-k+1)-1}{\lambda} .\end{array}\right.$

Now, if we apply the impuls condition (2) with the assumption of right continuousness at $t=k$, we find the difference system (7). Hence, the proof is complete.

It is noted that under the following conditions the impulsive differential system (1)-(2) has a unique solution: $x_{1}(-1)=\eta_{-1}, \quad x_{2}(-1)=\mu_{-1}, \quad x_{1}(0)=\eta_{0}, \quad x_{2}(0)=\mu_{0}$,

where $\eta_{-1}, \mu_{-1}, \eta_{0}$ and $\mu_{0}$ are real constants. Also, we note that under the same conditions the difference system (7) has a unique solution.

Theorem 4 Let $\left(x_{1}(t), x_{2}(t)\right)$ be a solution of (1)-(2). If $\left(x_{1}(k), x_{2}(k)\right)$ satisfies system (7) such that $\left(x_{1}(k+\right.$ $\left.p), x_{2}(k+p)\right)=\left(x_{1}(k), x_{2}(k)\right)$ for all $k \in \mathbb{N}$, then we have $\left(x_{1}(t+p), x_{2}(t+p)\right)=\left(x_{1}(t), x_{2}(t)\right)$ for all $t \in[0, \infty)$, where $p$ is the least positive integer.

Proof From (6), in the interval $k<t<k+1$, we have

$$
\begin{aligned}
x_{1}(t+p)= & x_{1}(k+p) \exp \lambda(t-k) \\
& -g\left(x_{2}(k+p-1)\right) \frac{\exp \lambda(t-k)-1}{\lambda} \\
= & x_{1}(k) \exp \lambda(t-k)-g\left(x_{2}(k-1)\right) \frac{\exp \lambda(t-k)-1}{\lambda} \\
= & x_{1}(t), \\
x_{2}(t+p)= & x_{2}(k+p) \exp \lambda(t-k) \\
& -g\left(x_{1}(k+p-1)\right) \frac{\exp \lambda(t-k)-1}{\lambda} \\
= & x_{2}(k) \exp \lambda(t-k)-g\left(x_{1}(k-1)\right) \frac{\exp \lambda(t-k)-1}{\lambda} \\
= & x_{2}(t) .
\end{aligned}
$$

Hence, the proof is complete. 
Theorem 5 Assume that $\lambda>0$ is a sufficiently small real constant. If $g$ is an odd function and there is a number $a>0$ such that

$g(a)=-a(d+1)$

and

$g^{\prime}(a) \neq-d-1, \quad 1-d, \quad d-1, \quad d+1$,

then there exists a solution $\left(x_{1}(t), x_{2}(t)\right)$ with least period 2 of (1)-(2).

Proof A solution $\left(x_{1}(t), x_{2}(t)\right)$ of (1)-(2) is given by (6). According to Theorem 4 , it comes out

$x_{1}(t+2)=x_{1}(t)$ and $\mathrm{x}_{2}(\mathrm{t}+2)=\mathrm{x}_{2}(\mathrm{t}) \quad$ for all $\mathrm{t} \in[0, \infty)$

provided that

$x_{1}(k+2)=x_{1}(k)$ and $\mathrm{x}_{2}(\mathrm{k}+2)=\mathrm{x}_{2}(\mathrm{k})$ for all $\mathrm{k} \in \mathbb{N}$,

where $\left(x_{1}(k), x_{2}(k)\right)$ is a solution of (7). So, we should only prove that (12) is true. Due to Theorem 1, we can choose a solution of (7) as

$$
\begin{aligned}
x_{1}(k)= & a_{0}(\alpha)+a_{1}(\alpha) \cos \pi k \text { and } \mathrm{x}_{2}(\mathrm{k})=\widetilde{a_{0}}(\alpha) \\
& +\widetilde{a_{1}}(\alpha) \cos \pi k,
\end{aligned}
$$

where $\alpha=e^{\lambda}, a_{i}$ and $\tilde{a}_{i}, i=0,1$ are real-valued functions. Substituting (13) into (7), we obtain

$$
\begin{aligned}
& a_{0}\left(1-\frac{\alpha}{d}\right)-a_{1}\left(1+\frac{\alpha}{d}\right) \cos \pi k+\frac{\beta}{d} g\left(\widetilde{a_{0}}-\widetilde{a_{1}} \cos \pi k\right)=0, \\
& \widetilde{a_{0}}\left(1-\frac{\alpha}{d}\right)-\widetilde{a_{1}}\left(1+\frac{\alpha}{d}\right) \cos \pi k+\frac{\beta}{d} g\left(a_{0}-a_{1} \cos \pi k\right)=0 .
\end{aligned}
$$

If system (14) is satisfied for $k=0$ and $k=1$, then it holds for all $k \in \mathbb{N}$. So, putting $k=0$ and $k=1$ into (14), we get the following system:

$$
\begin{aligned}
& a_{0}\left(1-\frac{\alpha}{d}\right)-a_{1}\left(1+\frac{\alpha}{d}\right)+\frac{\beta}{d} g\left(\widetilde{a_{0}}-\widetilde{a_{1}}\right)=0, \\
& \widetilde{a_{0}}\left(1-\frac{\alpha}{d}\right)-\widetilde{a_{1}}\left(1+\frac{\alpha}{d}\right)+\frac{\beta}{d} g\left(a_{0}-a_{1}\right)=0, \\
& a_{0}\left(1-\frac{\alpha}{d}\right)+a_{1}\left(1+\frac{\alpha}{d}\right)+\frac{\beta}{d} g\left(\widetilde{a_{0}}+\widetilde{a_{1}}\right)=0, \\
& \widetilde{a_{0}}\left(1-\frac{\alpha}{d}\right)+\widetilde{a_{1}}\left(1+\frac{\alpha}{d}\right)+\frac{\beta}{d} g\left(a_{0}+a_{1}\right)=0 .
\end{aligned}
$$

For $\lambda=0$, it is $\alpha=1$ and also $\beta(1)=1$. Hence, (15) reduces to the system

$$
\begin{aligned}
& a_{0}\left(1-\frac{1}{d}\right)-a_{1}\left(1+\frac{1}{d}\right)+\frac{1}{d} g\left(\widetilde{a_{0}}-\widetilde{a_{1}}\right)=0, \\
& \widetilde{a_{0}}\left(1-\frac{1}{d}\right)-\widetilde{a_{1}}\left(1+\frac{1}{d}\right)+\frac{1}{d} g\left(a_{0}-a_{1}\right)=0, \\
& a_{0}\left(1-\frac{1}{d}\right)+a_{1}\left(1+\frac{1}{d}\right)+\frac{1}{d} g\left(\widetilde{a_{0}}+\widetilde{a_{1}}\right)=0, \\
& \widetilde{a_{0}}\left(1-\frac{1}{d}\right)+\widetilde{a_{1}}\left(1+\frac{1}{d}\right)+\frac{1}{d} g\left(a_{0}+a_{1}\right)=0 .
\end{aligned}
$$

Since $g$ is odd and satisfies (9), system (16) has a solution as $a_{0}=\widetilde{a_{0}}=0, a_{1}=\tilde{a_{1}}=a$. Therefore, system (7) has a periodic solution with period 2 as

$x_{1}(k)=a \cos \pi k \quad$ and $\quad \mathrm{x}_{2}(\mathrm{k})=\mathrm{a} \cos \pi \mathrm{k}$.

This means that (1)-(2) has a solution of least period 2 for $\lambda=0$.

Now, let $\lambda$ be sufficiently small.

Again, we should establish a solution $\left(x_{1}(k), x_{2}(k)\right)$ of system (7) as in the form of (13). To fulfill this, we use the Implicit Function Theorem to show that there exists a $\delta>0$ such that there are functions $a_{0}(\alpha), \widetilde{a_{0}}(\alpha), a_{1}(\alpha)$ and $\widetilde{a_{1}}(\alpha)$ which are continuous for $0 \leq \alpha-1<\delta$ and $a_{0}(1)=$ $\widetilde{a_{0}}(1)=0, a_{1}(1)=\widetilde{a_{1}}(1)=a$. Putting (13) into (7), we find

$$
\begin{aligned}
& a_{0}(\alpha)\left(1-\frac{\alpha}{d}\right)-a_{1}(\alpha)\left(1+\frac{\alpha}{d}\right) \cos \pi k+\frac{\beta(\alpha)}{d} g\left(\widetilde{a_{0}}(\alpha)\right. \\
& \left.\quad-\widetilde{a_{1}}(\alpha) \cos \pi k\right)=0, \\
& \widetilde{a_{0}}(\alpha)\left(1-\frac{\alpha}{d}\right)-\widetilde{a_{1}}(\alpha)\left(1+\frac{\alpha}{d}\right) \cos \pi k+\frac{\beta(\alpha)}{d} g\left(a_{0}(\alpha)\right. \\
& \left.\quad-a_{1}(\alpha) \cos \pi k\right)=0 .
\end{aligned}
$$

Again, if this system holds for $k=0$ and $k=1$, then it will be satisfied for all $k \in \mathbb{N}$. Substituting $k=0$ and $k=1$ into (18), respectively, we obtain the system

$$
\begin{aligned}
& a_{0}(\alpha)\left(1-\frac{\alpha}{d}\right)-a_{1}(\alpha)\left(1+\frac{\alpha}{d}\right)+\frac{\beta(\alpha)}{d} g\left(\widetilde{a_{0}}(\alpha)-\widetilde{a_{1}}(\alpha)\right)=0, \\
& \widetilde{a_{0}}(\alpha)\left(1-\frac{\alpha}{d}\right)-\widetilde{a_{1}}(\alpha)\left(1+\frac{\alpha}{d}\right)+\frac{\beta(\alpha)}{d} g\left(a_{0}(\alpha)-a_{1}(\alpha)\right)=0, \\
& a_{0}(\alpha)\left(1-\frac{\alpha}{d}\right)+a_{1}(\alpha)\left(1+\frac{\alpha}{d}\right)+\frac{\beta(\alpha)}{d} g\left(\widetilde{a_{0}}(\alpha)+\widetilde{a_{1}}(\alpha)\right)=0, \\
& \widetilde{a_{0}}(\alpha)\left(1-\frac{\alpha}{d}\right)+\widetilde{a_{1}}(\alpha)\left(1+\frac{\alpha}{d}\right)+\frac{\beta(\alpha)}{d} g\left(a_{0}(\alpha)+a_{1}(\alpha)\right)=0 .
\end{aligned}
$$

Since $g$ is odd, the Jacobian determinant of system (19) at $\alpha=1$ is

$$
\begin{aligned}
J= & \frac{1}{d^{4}}\left|\begin{array}{cccc}
d-1 & -d-1 & g^{\prime}(a) & -g^{\prime}(a) \\
g^{\prime}(a) & -g^{\prime}(a) & d-1 & -d-1 \\
d-1 & d+1 & g^{\prime}(a) & g^{\prime}(a) \\
g^{\prime}(a) & g^{\prime}(a) & d-1 & d+1
\end{array}\right| \\
= & \frac{1}{d^{4}}\left(-4 d^{4}+8 d^{2} g^{\prime 2}(a)+8 d^{2}-4 g^{\prime 4}(a)+8 g^{\prime 2}(a)-4\right) \\
= & \frac{-4}{d^{4}}\left(d-g^{\prime}(a)-1\right)\left(d-g^{\prime}(a)+1\right)\left(d+g^{\prime}(a)-1\right) \\
& \times\left(d+g^{\prime}(a)+1\right) .
\end{aligned}
$$

From (10), we obtain that $J \neq 0$ at $\alpha=1$. 
So, for sufficiently small $\lambda>0$, there is a $\delta>0$ such that there exist functions $a_{0}(\alpha), \widetilde{a_{0}}(\alpha), a_{1}(\alpha), \widetilde{a_{1}}(\alpha)$ that are continuous on $[1,1+\delta)$ and form a solution of system (19) such that $a_{0}(1)=\widetilde{a_{0}}(1)=0, a_{1}(1)=\widetilde{a_{1}}(1)=a$.

Hence, the proof is complete.

Remark 6 If $d=1$, then the impulsive differential system with piecewise constant arguments (1)-(2) reduces to the continuous system

$x_{1}^{\prime}(t)=\lambda x_{1}(t)-g\left(x_{2}[t-1]\right)$,

$x_{2}^{\prime}(t)=\lambda x_{2}(t)-g\left(x_{1}[t-1]\right), \quad 0 \leq t<\infty$.

In this case, Theorem 3,4 and 5 are still valid for $d=1$.

Open Access This article is distributed under the terms of the Creative Commons Attribution License which permits any use, distribution, and reproduction in any medium, provided the original author(s) and the source are credited.

\section{References}

1. Bainov, D.D., Simeonov, P.S.: Impulsive Differential Equations: Periodic Solutions and Applications. Longman Scientific and Technical, Harlow (1993)

2. Samoilenko, A.M., Perestyuk, N.A.: Impulsive Differential Equations. World Scientific, Singapore (1995)

3. Lakshmikantham, V., Bainov, D.D., Simeonov, P.S.: Theory of Impulsive Differential Equations. World Scientific, Singapore (1998)

4. Bereketoglu, H., Karakoc, F.: Asymptotic constancy for impulsive delay differential equations. Dyn. Syst. Appl. 17, 71-84 (2008)

5. Bereketoglu, H., Pituk, M.: Asymptotic constancy for nonhomogeneous linear differential equations with unbounded delay. Discrete Contin. Dyn. Syst., 100-107 (2003)

6. De la Sen, M.: Stability of impulsive time-varying systems and compactness of the operators mapping the input space into the state and output spaces. J. Math. Anal. Appl. 321, 621-650 (2006)

7. Luo, Z., Luo, Y.: Asymptotic stability for impulsive functional differential equations. Appl. Math. Mech. 30(10), 1317-1324 (2009)

8. Zhang, Y., Zhao, A., Yan, J.: Oscillation criteria for impulsive delay differential equations. J. Math. Anal. Appl. 205(2), 461-470 (1997)

9. Busenberg, S., Cooke, K.L.: Models of vertically transmitted diseaseases with sequential-continuous dynamics. In:
Lakshmikantham, V. (Ed.) Nonlinear Phenomena in Mathematical Sciences, pp 179-187. Academic Press, London (1982)

10. Cooke, K.L., Wiener, J.: Retarded differential equations with piecewise constant delays. J. Math. Anal. Appl. 99(1), 265-297 (1984)

11. Aftabizadeh, A.R., Wiener, J.: Oscillatory and periodic solutions for systems of two first order linear differential equations with piecewise constant argument. Appl. Anal. 26(4), 327-333 (1988)

12. Aftabizadeh, A.R., Wiener, J., Xu, J.M.: Oscillatory and periodic solutions of delay differential equations with piecewise constant argument. Proc. Am. Math. Soc. 99(4), 673-679 (1987)

13. Györi, I.: On the approximation of the solutions of delay differential equations by using piecewise constant arguments. Internat. J. Math. Math. Sci. 14(1), 111-126 (1991)

14. Győri, I., Ladas, G.: Linearized oscillations for equations with piecewise constant argument. Differ. Integr. Equ. 2, 123-131 (1989)

15. Huang, Y.K.: Oscillations and asymptotic stability of solutions of first order neutral differential equations with piecewise constant argument. J. Math. Anal. Appl. 149(1), 70-85 (1990)

16. Liang, H., Wang, G.: Existence and uniqueness of periodic solutions for a delay differential equation with piecewise constant arguments. Port. Math. 66(1), 1-12 (2009)

17. Muroya, Y.: New contractivity condition in a population model with piecewise constant arguments. J. Math. Anal. Appl. 346(1), 65-81 (2008)

18. Pinto, M.: Asymptotic equivalence of nonlinear and quasilinear differential equations with piecewise constant arguments. Math. Comput. Modelling 49(9-10), 1750-1758 (2009)

19. Yuan, R.: The existence of almost periodic solutions of retarded differential equations with piecewise constant argument. Nonlinear Anal. 48(7), 1013-1032 (2002)

20. Wiener, J.: Generalized Solutions of Functional Differential Equations. World Scientific, Singapore (1994)

21. Wiener, J., Lakshmikantham, V.: Differential equations with piecewise constant argument and impulsive equations. Nonlinear Stud. 7, 60-69 (2000)

22. Luo, M.H.: Existence of periodic solutions to impulsive differential equations with piecewise constant argument. (Chinese) Math. Theory Appl. (Chansha), 25 (2005)

23. Karakoc, F., Bereketoglu, H., Seyhan, G.: Oscillatory and periodic solutions of impulsive differential equations with piecewise constant argument. Acta Appl. Math. 110(1), 499-510 (2010)

24. Seifert, G.: Periodic solutions of certain hybrid delay-differential equations and their corresponding difference equations. J. Differ. Equ. Appl. 14, 295-299 (2008)

25. Elaydi, S.N.: Discrete Chaos: With Applications in Science and Engineering. Second edn. Chapman \& Hall/CRC, Boca Raton (2008, ISBN: 978-1-58488-592-4) 